\title{
Simultaneous pulse wave and flow estimation at high-framerate using plane wave and transverse oscillation on carotid phantom
}

\author{
Vincent Perrot, Lorena Petrusca, Adeline Bernard, Didier Vray and Hervé Liebgott \\ Univ. Lyon, INSA-Lyon, UCBL, UJM-Saint-Étienne, CNRS, Inserm, CREATIS UMR 5220, U1206, F-69621 \\ Lyon, France \\ vincent.perrot@creatis.insa-lyon.fr
}

\begin{abstract}
In this paper a global estimation method based on transverse oscillation to simultaneously extract wall and flow velocities at high-framerate is presented. Several carotid phantoms with various parameters were made to validate the method. All acquisitions were performed at high-framerate $(7500$ images per second) using horizontal plane wave with a 3 cycles sinusoidal transmit pulse. Transverse oscillation was introduced in post-acquisition. Finally, velocity vectors were extracted thanks to a phase based estimator with a region of interest of $2 \mathrm{~mm}(8$ axial wavelengths) per $2.96 \mathrm{~mm}$ (2 lateral wavelengths) for each pixel. Results are promising, all standard deviations are lower than $10 \%$ and the method is now validated for a deeper study. Indeed, flow and pulse wave velocities computed by the algorithm are in accordance with pressure columns and number of freezethaw cycles.
\end{abstract}

Index Terms-pulse wave, flow, transverse oscillation, plane wave, motion estimation, flow estimation, carotid phantom

\section{INTRODUCTION}

Ultrafast or high-framerate ultrasound imaging is an imaging modality which has been developed over the last fifteen years and which has found numerous applications [1]. Indeed, plane wave imaging allows a high-framerate of more than 1000 images per second (according to depth of interest) against 150 images per second at most for conventional imaging [2]. This increased framerate is useful to image fast phenomena of tissues [3], [4]. Plane wave imaging is used for artery elasticity assessment as well as flow estimation [5], [6].

With the purpose of evaluating the healthy or pathological state of blood vessels, one usual method consists to measure several parameters linked to hemodynamic (flow) [7] or to mechanical parameters of arterial wall (wall thickness, distensibility, pulse wave velocity) [8]. These two aspects are generally assessed using two different ultrasound sequences

The Verasonics system was cofounded by the FEDER program, SaintEtienne Metropole (SME) and Conseil General de la Loire (CG42) within the framework of the SonoCardioProtection Project leaded by Pr Pierre Croisille and Dr Magalie Viallon as principal investigators. This work was also performed within the framework of the LABEX CELYA (ANR-10-LABX-0060) and LABEX PRIMES (ANR-10-LABX-0063) of Université de Lyon, within the program "Investissements d'Avenir" (ANR-11-IDEX-0007) operated by the French National Research Agency (ANR). and processing techniques. This can be explained by the difference in terms of signal intensity between the tissue and blood (more than $40 \mathrm{~dB}$ ). But also the nature of blood and wall's motion is different in terms of direction (along the vessel for flow and perpendicular to the vessel for pulse wave) and velocity. However, wall and flow are naturally linked and should be analyzed together. In order to better evaluate and understand blood-wall interaction, an imaging technique able to provide simultaneously both characteristics would be highly relevant.

Transverse oscillation has been developed for flow estimation and provides 2D velocity estimation [9], [10]; our group was among the first to propose a method combining the transmission of plane wave with transverse oscillation for flow imaging [11]. Moreover, the method has been adapted for tissue motion estimation [12], [13]. Consequently, we propose to use transverse oscillation technique to simultaneously extract flow and wall motion at high-framerate using one unique plane wave sequence.

The aim of this paper is to show the feasibility of the method with a carotid phantom (PVA) filled with a blood-mimicking fluid (Orgasol ${ }^{\circledR}$ ). Different set-ups in terms of pressure (flow) and stiffness (wall) have been investigated. In the following, section II introduces the experimental set-up, the material and the adapted transverse oscillation method for simultaneous blood and wall motion imaging. Then section III presents the results. Finally, section IV concludes the paper with a brief summary of the method and results.

\section{MATERIAL AND METHOD}

\section{A. Experimental set-up}

Several PVA phantoms have been molded (Tab. I) for the experiments in order to mimic carotid artery, and an Orgasol $^{\circledR}$ solution has been used to mimic blood. The phantom preparation was composed of PVA, silica and distilled water (10\%,1\% and $89 \%$ in weight, respectively). PVA acquires its properties by freeze-thaw cycles process; the higher the number of cycles, the higher the Young's modulus. PVA ensures elasticity while silica provides 
scatterers in vessel wall. The resulting phantoms are very close to biological tissues in terms of acoustic and mechanical characteristics [14]. Finally, phantoms with four different number of freeze-thaw cycles (from 2 to 5 cycles) have been investigated (Tab. I). For each number of cycles two samples have been produced. The blood mimicking fluid was made with $5 \mu \mathrm{m} \mathrm{Orgasol}{ }^{\circledR}$, glycerol, surfactant and distilled water ( $2 \%, 10 \%, 1 \%$ and $87 \%$ in weight, respectively). Orgasol ${ }^{\circledR}$ provides scatterers, glycerol sets viscosity and surfactant ensures that the scattering particles are dispersed in the fluid. The resulting blood-mimicking fluid is in accordance with the standard physical and acoustic properties of blood [15].

TABLE I

EXPERIMENTAL CONDITIONS

\begin{tabular}{|c|c|}
\hline Phantom length & $8 \mathrm{~cm}$ \\
\hline Phantom internal diameter & $8 \mathrm{~mm}$ \\
\hline Phantom thickness & $2 \mathrm{~mm}$ \\
\hline Valve opening duration & $300 \mathrm{~ms}$ \\
\hline Number of freeze-thaw cycles & $2,3,4,5$ \\
\hline Column height & $25 \mathrm{~cm}, 45 \mathrm{~cm}$ \\
\hline
\end{tabular}

The inlet of the phantom is connected to a fluid column (filled with the blood-mimicking fluid) while the output is connected to an open tank (at the ambient pressure of the experiment room) and the phantom is immersed in water for ultrasound measurement. A solenoid-valve, controlled by a function generator is placed between the inlet and the pressure column to set the flow cycles (Tab. I). The experimental set-up is idle before each acquisition and experiment.

\section{B. Data acquisition}

TABLE II

ACQUISITION PARAMETERS

\begin{tabular}{|c|c|}
\hline Probe pitch & $296 \mu \mathrm{m}$ \\
\hline Probe number of elements & 128 \\
\hline Excitation pulse & 3 cycles at $6.25 \mathrm{MHz}$ \\
\hline Sampling frequency & $25 \mathrm{MHz}$ \\
\hline Speed of sound & $1540 \mathrm{~m} / \mathrm{s}$ \\
\hline Pulse repetition frequency & $7500 \mathrm{~Hz}$ \\
\hline Compounding & No \\
\hline Steering angle & $90^{\circ}$ \\
\hline Transmit apodization & Hanning \\
\hline Receive apodization & Rectangular \\
\hline
\end{tabular}

In this study, a Verasonics ultrasound system (Verasonics Inc., Redmond, WA) with the L7-4 probe were used; acquisition parameters are described in Tab. II. Acquisitions were performed with plane wave; only one horizontal plane wave was transmitted in order to achieve an ultrafast imaging at high-framerate (no compounding). The ultrasound probe was immersed in water and placed parallel to the superior wall at a depth of $6 \mathrm{~mm}$. The Verasonics system was triggered by the function generator to start acquisition $100 \mathrm{~ms}$ before the activation of the solenoid valve which remains opened during $300 \mathrm{~ms}$ and data were acquired during a total time of 1.2 s. For each configuration (phantom, number of freeze-thaw cycles and column height) 3 acquisitions were performed resulting in 48 acquisitions in total. The reconstruction was done in postacquisition using the Stolt's migration technique performed in the Fourier domain [16].

\section{Flow and wall motion estimation}

Before flow and wall estimation a regression polynomial wall filter was applied on each pixel along time. The fitting polynomial was removed to keep the high-frequency components only [17]. Motion (wall and flow) estimation technique is based on transverse oscillation technique.

In this work, the implementation of transverse oscillation was performed in post-acquisition in the Fourier domain [18]; this method consists to multiply the 2D Fourier spectrum of each RF image to only keep the desired lateral wavelength. The resulting Fourier spectrum is composed of "four" spots corresponding to the axial (natural) and lateral (introduced with the mask multiplication) frequencies. Once the image has been filtered with this approach it becomes possible to extract the 2D motion vector map using the phase-based technique described in [19]. Transverse oscillation was introduced with a wavelength of $1.48 \mathrm{~mm}$, the natural oscillation due to ultrasound along the axial axis has a wavelength of $0.25 \mathrm{~mm}$. Motion was computed between each successive frame through a block of $2 \mathrm{~mm}$ (8 axial wavelengths) per $2.96 \mathrm{~mm}$ (2 lateral wavelengths). After estimation, velocities are filtered by a mean temporal filter of $25 \mathrm{~ms}$. For displaying the results, flow velocity is only showed on the central part of the probe (width of $2 \mathrm{~cm}$ ) due to a lack of signal near the image edges. However, wall velocity is completely displayed because the intensity signal is sufficient in the wall.

\section{RESULTS}

\section{A. Flow and pulse wave visualization}

The main interest of the method is to directly quantify and visualize flow and pulse wave simultaneously. An example of this kind of visualization is provided (Fig. 1) for one phantom of this study.

The wall and flow have different properties. Concerning the velocities, the wall is subject to a maximum velocity of $1 \mathrm{~mm} / \mathrm{s}$ (Fig. 1 (a), (b), (c) and (d)) while the maximum flow velocity is around $0.3 \mathrm{~m} / \mathrm{s}$ (Fig. 1 (d)). Moreover, wall motion is estimated from the axial axis whereas flow is extracted from the lateral axis. More importantly, the maximum wall velocity and the maximum flow velocity are delayed in time. In fact, the maximum wall velocity can be seen at the beginning of the acquisition when the pulse wave crosses the phantom (Fig. 1 (a), (b) and (c)) between $117.33 \mathrm{~ms}$ to $120 \mathrm{~ms}$ from left to right. During the propagation of the pulse wave the flow is close to zero. Indeed, the maximum estimated velocity profile is noticeable at $333.33 \mathrm{~ms}$ (Fig. 1 (d)) and when the flow is maximum the wall velocity is minimum. The difference of signal between the flow and the wall was measured to 


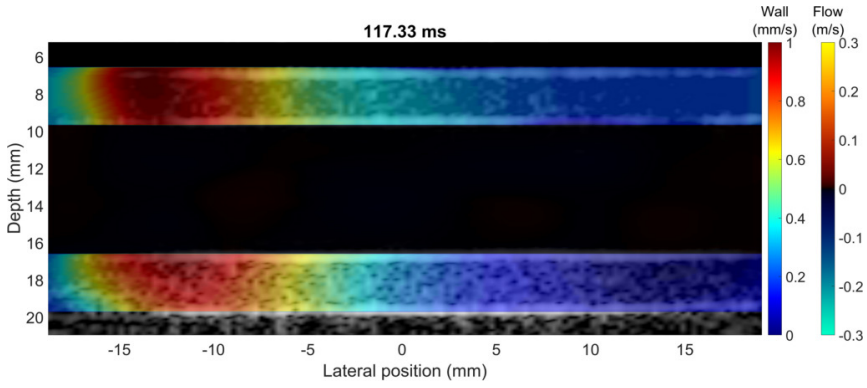

(a)

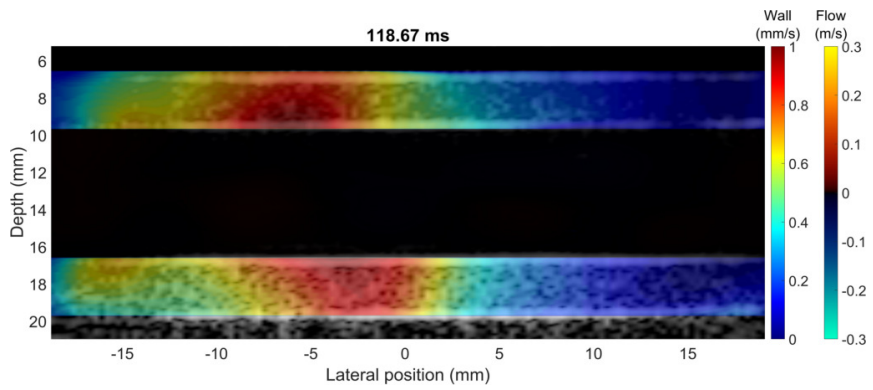

(b)

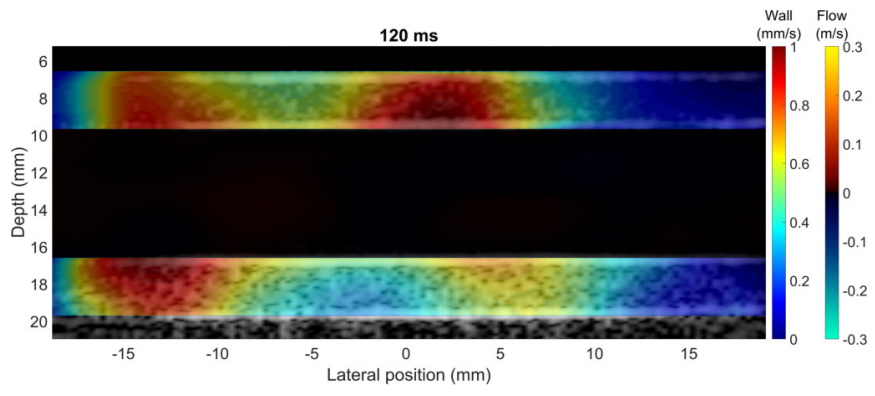

(c)

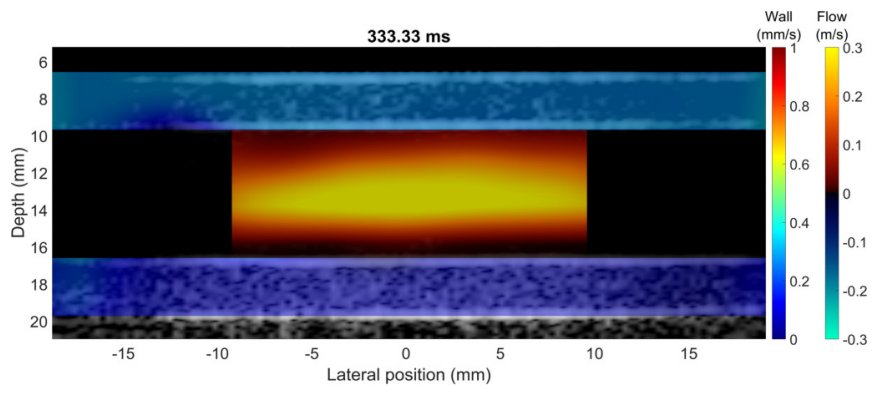

(d)

Fig. 1. Four snapshots of the wall and flow velocities at $117.33 \mathrm{~ms}$ (a), $118.67 \mathrm{~ms}$ (b), $120 \mathrm{~ms}$ (c) and $333.33 \mathrm{~ms}$ (d) after the beginning of an acquisition. Wall velocity is expressed in $\mathrm{mm} / \mathrm{s}$ while flow velocity is displayed in $\mathrm{m} / \mathrm{s}$ only on the center part of the lumen. (a), (b) and (c) show the pulse wave arriving from the left part of the phantom. (d) corresponds to the time when the flow is maximum at the center of the lumen.

approximately $40 \mathrm{~dB}$ (center of the lumen and distal wall). Consequently, the wall intensity is more important than flow intensity and a gap in terms of signal is present as is the case for real carotid data. The algorithm is able to extract a large range of velocities which is vital for simultaneously quantifying wall and flow. In addition, the method is able to extract vector velocities as required for wall and flow study.

\section{B. Flow according to pressure}

Flow has been estimated for two different pressure columns $(25 \mathrm{~cm}$ and $45 \mathrm{~cm})$, the resulting profiles across the vessel (average and standard deviation) are shown below (Fig. 2).

Profiles are quasi-parabolic with a maximum value of $0.399 \mathrm{~m} / \mathrm{s} \pm 6.5 \%$ in high pressure and $0.327 \mathrm{~m} / \mathrm{s} \pm 4.0 \%$ in low pressure. Maximum flow velocity decreased as expected with input pressure and the standard deviations remain low in comparison with the maximum values.

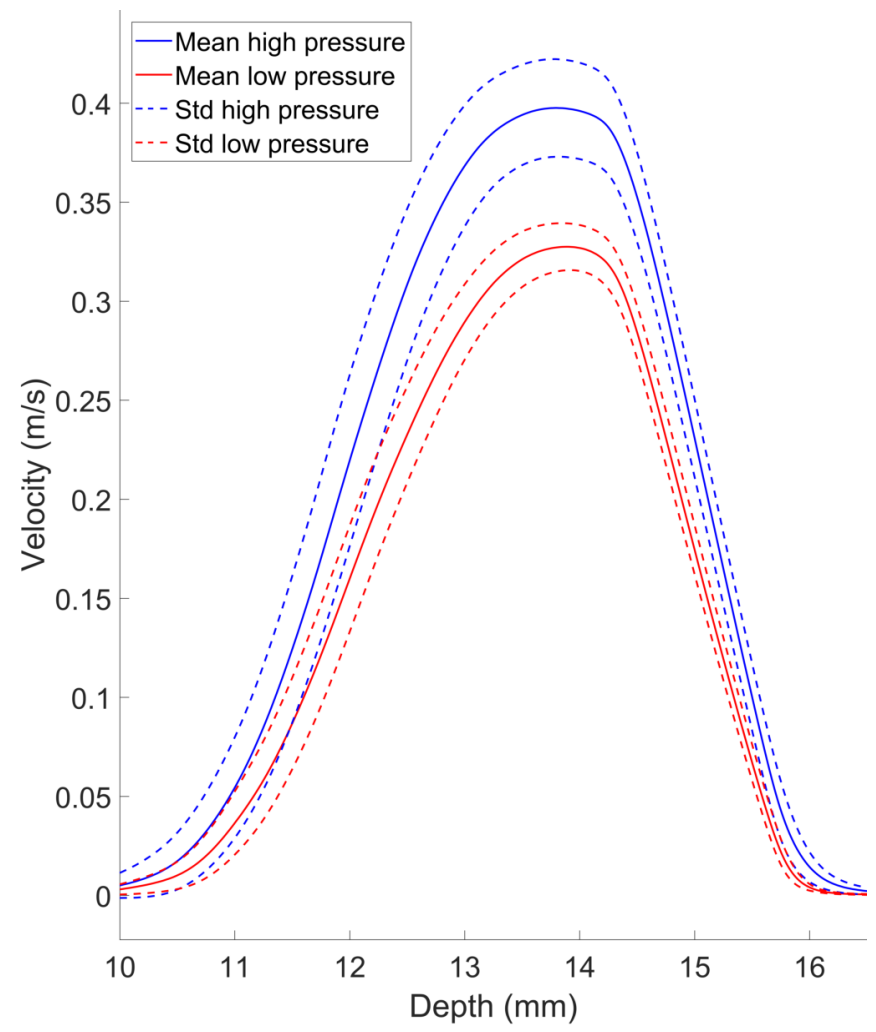

Fig. 2. Estimated velocity profiles on average (with all phantoms from 2 to 5 cycles) for the high pressure (blue) and the low pressure (red) at $333.33 \mathrm{~ms}$.

\section{Pulse wave velocity according to elasticity}

Pulse wave velocity can be extracted from wall velocity maps by tracking the propagation of the wall velocity front (Fig. 1 (a), (b) and (c)) along time. The position of the propagating pulse wave was defined where the wall acceleration attains its maximum and correspond to the foot of the pulse wave. The results for each number of freeze-thaw cycles (on average with standard deviation) are displayed (Fig. 3).

The pulse wave velocity increases with the number of freeze-thaw cycles as predicted from $2.21 \mathrm{~m} / \mathrm{s} \pm 1.78 \%$ to $4.89 \mathrm{~m} / \mathrm{s} \pm 7.63 \%$. Moreover, the shape of the curve matches with our expectations. Indeed, the theoretical curve 


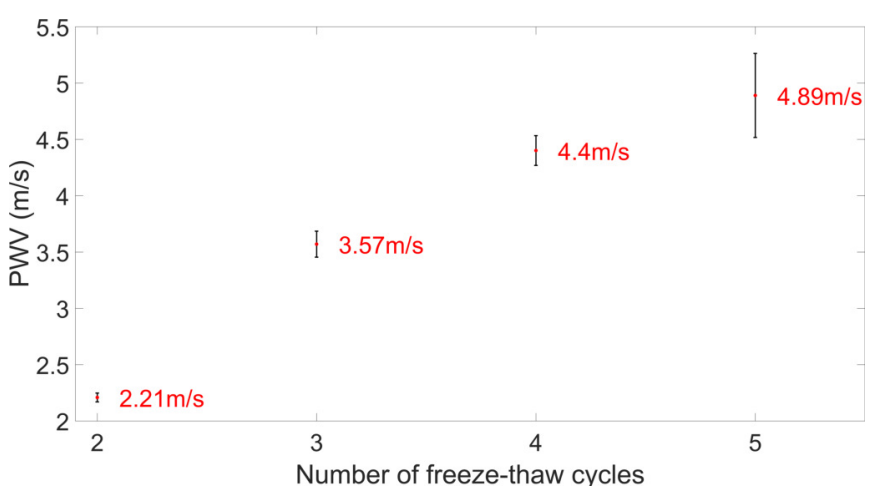

Fig. 3. Estimated pulse wave velocities on average depending on freeze-thaw cycles.

converges after 5 freeze-thaw cycles. The standard deviations remain small in regard to the pulse wave velocities even if they increase with the number of freeze-thaw cycles. The first explanation is that the number of frames where the pulse wave velocity is propagating decrease with the increase of the pulse wave velocity which causes a loss of robustness. Secondly, the phantoms were made in a month and in several steps. Consequently, a few differences during the thawing process might have occurred in particular some temperature variation. Nevertheless, the method is still able to extract pulse wave velocity in accordance with the elasticity of the medium.

\section{SUMMARY AND CONCLUSION}

In this paper, an imaging method able to provide simultaneously information about pulse wave propagation and blood flow has been proposed and investigated. It is based on transverse oscillation with horizontal plane wave insonification.

The algorithm is able to estimate the velocity vector from flow (lateral) and wall (axial). The validation process has been performed on a total of 48 plane wave acquisitions composed with 8 phantoms with various stiffnesses and flow velocities. Pulse wave velocities were computed from $2.21 \mathrm{~m} / \mathrm{s}$ to $4.89 \mathrm{~m} / \mathrm{s}$ and estimated flow velocities are $0.399 \mathrm{~m} / \mathrm{s}$ and $0.327 \mathrm{~m} / \mathrm{s}$; all standard deviations are lower than $10 \%$. Results show that the technique allows a simultaneous study and visualization of flow and wall motion. Moreover, fastest phenomena like pulse wave can be extracted after computation. The estimated flows and pulse wave velocities are in accordance with the applied pressure columns and stiffnesses, respectively, which validate the feasibility of our approach.

\section{REFERENCES}

[1] M. Tanter and M. Fink, "Ultrafast imaging in biomedical ultrasound," IEEE Transactions on Ultrasonics, Ferroelectrics, and Frequency Control, vol. 61, no. 1, pp. 102-119, jan 2014.

[2] D. Vray, E. Brusseau, V. Detti, F. Varray, A. Basarab, O. Beuf, O. Basset, C. Cachard, H. Liebgott, and P. Delachartre, "Ultrasound medical imaging," in Medical Imaging Based on Magnetic Fields and Ultrasounds. John Wiley \& Sons, Inc., mar 2014, pp. 1-72.
[3] J. Vappou, J. Luo, and E. E. Konofagou, "Pulse wave imaging for noninvasive and quantitative measurement of arterial stiffness in vivo," American Journal of Hypertension, vol. 23, no. 4, pp. 393-398, apr 2010.

[4] T. Mirault, M. Pernot, M. Frank, M. Couade, R. Niarra, M. Azizi, J. Emmerich, X. Jeunemaître, M. Fink, M. Tanter, and E. Messas, "Carotid stiffness change over the cardiac cycle by ultrafast ultrasound imaging in healthy volunteers and vascular ehlers-danlos syndrome," Journal of Hypertension, vol. 33, no. 9, pp. 1890-1896, sep 2015.

[5] J. Udesen, F. Gran, K. Hansen, J. Jensen, C. Thomsen, and M. Nielsen, "High frame-rate blood vector velocity imaging using plane waves: Simulations and preliminary experiments," IEEE Transactions on Ultrasonics, Ferroelectrics and Frequency Control, vol. 55, no. 8, pp. 17291743, aug 2008.

[6] S. Fadnes, I. K. Ekroll, S. A. Nyrnes, H. Torp, and L. Lovstakken, "Robust angle-independent blood velocity estimation based on dual-angle plane wave imaging," IEEE Transactions on Ultrasonics, Ferroelectrics, and Frequency Control, vol. 62, no. 10, pp. 1757-1767, oct 2015.

[7] P. M. Hansen, J. B. Olesen, M. J. Pihl, T. Lange, S. Heerwagen, M. M. Pedersen, M. Rix, L. Lönn, J. A. Jensen, and M. B. Nielsen, "Volume flow in arteriovenous fistulas using vector velocity ultrasound," Ultrasound in Medicine \& Biology, vol. 40, no. 11, pp. 2707-2714, nov 2014.

[8] A. Bjallmark, B. Lind, M. Peolsson, K. Shahgaldi, L.-A. Brodin, and J. Nowak, "Ultrasonographic strain imaging is superior to conventional non-invasive measures of vascular stiffness in the detection of agedependent differences in the mechanical properties of the common carotid artery," European Journal of Echocardiography, vol. 11, no. 7, pp. 630-636, mar 2010.

[9] J. Jensen and P. Munk, "A new method for estimation of velocity vectors," IEEE Transactions on Ultrasonics, Ferroelectrics and Frequency Control, vol. 45, no. 3, pp. 837-851, may 1998.

[10] J. Jensen, "A new estimator for vector velocity estimation [medical ultrasonics]," IEEE Transactions on Ultrasonics, Ferroelectrics and Frequency Control, vol. 48, no. 4, pp. 886-894, jul 2001.

[11] M. Lenge, A. Ramalli, P. Tortoli, C. Cachard, and H. Liebgott, "Planewave transverse oscillation for high-frame-rate 2-d vector flow imaging," IEEE Transactions on Ultrasonics, Ferroelectrics, and Frequency Control, vol. 62, no. 12, pp. 2126-2137, dec 2015.

[12] H. Liebgott, A. Basarab, P. Gueth, D. Friboulet, and P. Delachartre, "Transverse oscillations for tissue motion estimation," Ultrasonics, vol. 50, no. 6, pp. 548-555, may 2010.

[13] S. Salles, A. J. Y. Chee, D. Garcia, A. C. H. Yu, D. Vray, and H. Liebgott, "2-d arterial wall motion imaging using ultrafast ultrasound and transverse oscillations," IEEE Transactions on Ultrasonics, Ferroelectrics, and Frequency Control, vol. 62, no. 6, pp. 1047-1058, jun 2015.

[14] J. Fromageau, J.-L. Gennisson, C. Schmitt, R. Maurice, R. Mongrain, and G. Cloutier, "Estimation of polyvinyl alcohol cryogel mechanical properties with four ultrasound elastography methods and comparison with gold standard testings," IEEE Transactions on Ultrasonics, Ferroelectrics and Frequency Control, vol. 54, no. 3, pp. 498-509, mar 2007.

[15] K. V. Ramnarine, D. K. Nassiri, P. R. Hoskins, and J. Lubbers, "Validation of a new blood-mimicking fluid for use in doppler flow test objects," Ultrasound in Medicine \& Biology, vol. 24, no. 3, pp. 451-459, mar 1998.

[16] D. Garcia, L. L. Tarnec, S. Muth, E. Montagnon, J. Poree, and G. Cloutier, "Stolt's f-k migration for plane wave ultrasound imaging," IEEE Transactions on Ultrasonics, Ferroelectrics, and Frequency Control, vol. 60, no. 9, pp. 1853-1867, sep 2013.

[17] S. Bjaerum, H. Torp, and K. Kristoffersen, "Clutter filter design for ultrasound color flow imaging," IEEE Transactions on Ultrasonics, Ferroelectrics and Frequency Control, vol. 49, no. 2, pp. 204-216, feb 2002.

[18] S. Salles, D. Garcia, B. Bou-Said, F. Savary, A. Serusclat, D. Vray, and H. Liebgott, "Plane wave transverse oscillation (PWTO): An ultra-fast transverse oscillation imaging mode performed in the fourier domain for $2 \mathrm{~d}$ motion estimation of the carotid artery," in 2014 IEEE 11th International Symposium on Biomedical Imaging (ISBI). IEEE, apr 2014.

[19] A. Basarab, P. Gueth, H. Liebgott, and P. Delachartre, "Phase-based block matching applied to motion estimation with unconventional beamforming strategies," IEEE Transactions on Ultrasonics, Ferroelectrics and Frequency Control, vol. 56, no. 5, pp. 945-957, may 2009. 